\title{
Control of automated systems with a structured light illumination source
}

\author{
Johannes Herrnsdorf, Michael J. Strain, and Martin D. Dawson \\ Institute of Photonics, Department of Physics, University of Strathclyde, Glasgow G1 1RD (UK), \\ Email: johannes.herrnsdorf@strath.ac.uk
}

\begin{abstract}
Autonomous mobile devices can self-locate, navigate and receive control signals using structured illumination from a light-emitting diode array. We present a prototype system and discuss the properties of the optical wireless control.
\end{abstract}

\section{INTRODUCTION}

Gallium Nitride light-emitting diodes (LEDs) are a key enabling technology for energy-efficient illumination that can be electronically interfaced and modulated at frequencies up to several hundreds of $\mathrm{MHz}$. These properties make them attractive as transmitters in high-bandwidth optical wireless networks with low energy consumption.

By synchronized modulation of a number of spatially distributed GaN LEDs, e.g. an array of LEDs, a range of powerful spatio-temporal encoding schemes are feasible. In a first instance, individual parallel network connections can be made to multiple receivers in the area [1], [2], or a receiver array can be used to enable $\mathrm{Gb} / \mathrm{s}$ multiple-input multipleoutput data transmission [3].

Here we employ GaN LED arrays in a format that is particularly suitable for application in the context of the internet of things, automated manufacturing and autonomous systems. The LED illumination source comprises an array of individually addressable LED elements [4], [5] which are mapped onto the illuminated area by imaging optics as illustrated in Fig. 1. Such a configuration can be used to provide a positioning system that minimises the complexity at the receiver end [2].

Our presentation discusses how such LED arrays can be used to implement a control system for autonomous devices in the illuminated area where the entire control of the device operation is realized through the illumination. Commands to the device as well as navigation aid are transmitted via timesequences of appropriately chosen illumination patterns.

\section{ELECTRONICALLY INTERFACED LED ARRAY SOURCES}

For our demonstrator system we use blue-emitting $16 \times 16$ GaN LED arrays that are bump-bonded to a receiving complementary metal-oxide-semiconductor (CMOS) driver chip [1], [2]. These devices can display pattern sequences at a rate of up to $2000 \mathrm{fps}$, limited by the driver electronics. The output from the array is projected to an $8 \times 8 \mathrm{~cm}$ area in which the autonomous device moves.

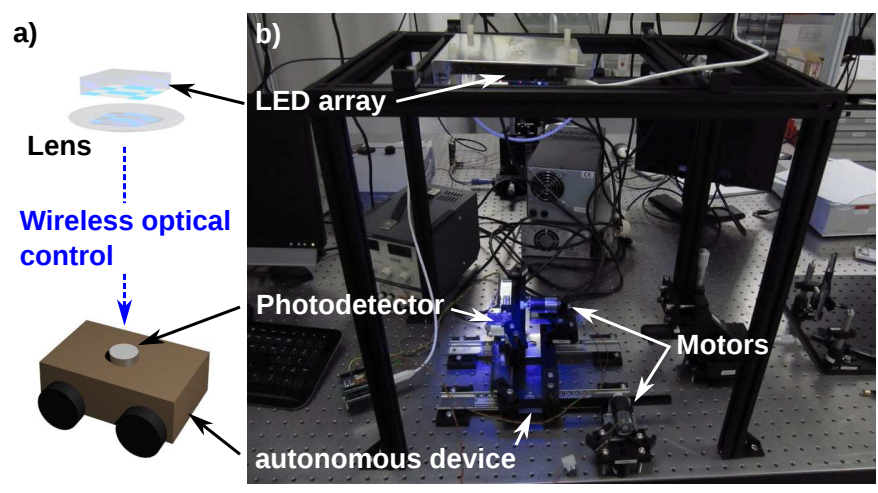

Fig. 1. a) Schematic of optical wireless control of an autonomous mobile device and $b$ ) photograph of the prototype demonstrator.

\section{ERROR TOLERANT PATTERN SEQUENCES FOR SPATIO-TEMPORAL ENCODING}

Both, data transmission and positioning of the autonomous device are realized through projection of a time-sequence of appropriate spatial illumination patterns. In particular, the patterns used as navigation aid need careful consideration. Our initial demonstration of such a system used illumination patterns adapted from imaging techniques, such as the Hadamard basis [6]. Subsequently, the position update rate was improved by optimising the pattern sequence towards minimal sequence length. With pattern sequences as short as $2 \log _{2} N$, where $N$ is the number of LED elements, full precision positioning is enabled while at the same time providing uniform flicker-free illumination of the scene.

Not only is the speed of positioning important, but also the reliability and robustness. The pattern sequence length can be traded off against the signal to noise ratio (SNR) and favorable behavior in case of a false reading. Here, we compare two types of pattern sequences, referred to as sequence type A with a length of $2 \log _{2} N$ and type B with a length of $2 \sqrt{N}$. Both sequences are illustrated on the example of an $8 \times 8$ array in Fig. 2a and $b$.

In either case, the optical signal at a given position within the area corresponds to a unique bit-sequence, where each bit is encoded in the differential signal of a pair of patterns that are inverse to each other. These pattern pairs are labeled in Fig. 2a and b as normal ("n") and inverse ("i") patterns. In the case of sequence $\mathrm{A}$, a device receiving this bit-sequence can determine its position in a binary search fashion. In the 


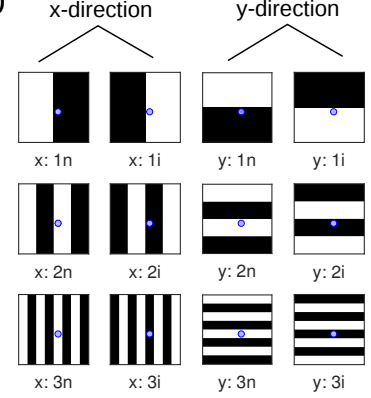

c)

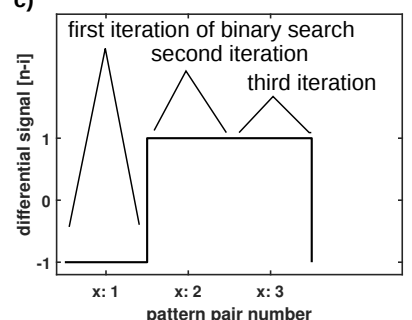

b)

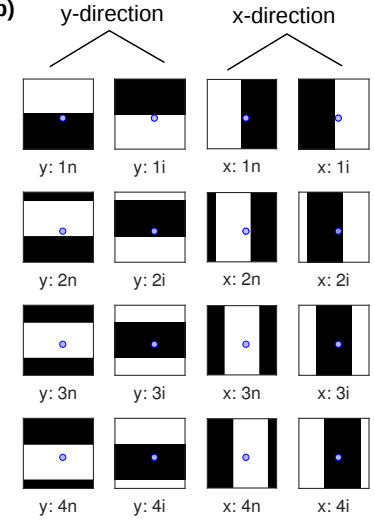

d)

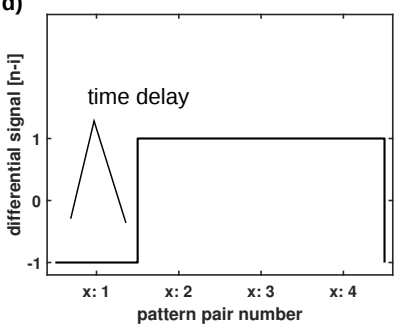

Fig. 2. Illustration of pattern sequences for position control: $a$ ) sequence type $\mathrm{A}$ and $b$ ) sequence type $\mathrm{B}$. The exemplary position of a photodetector placed at $x=5, y=5$ is indicated by a blue disk. The patterns are grouped in pairs that are inverse to each other, indicated by "n" (normal) and "i" (inverse). Equivalent pattern pairs are applied in $x$ - and $y$-direction. Subfigures $c$ ) and $d$ ) show schematically the differential signal received from two paired normalinverse patterns as a function of pattern pair number. For simplicity, only the patterns in $x$-direction are considered and the trace shown corresponds to the detector at $x=5$ indicated by the blue disk. $c$ ) corresponds to sequence A and $d$ ) to sequence $\mathrm{B}$.

case of sequence $\mathrm{B}$, the position of the detector corresponds to the time delay of a square wave signal in the bit-sequence with a period of $\sqrt{N}$ pattern pairs. The different encoding of the position information is illustrated in Fig. $2 \mathrm{c}$ and d. It is indicated how, in the case of sequence A, the position is given in three iterations of a binary search, whereas in the case of sequence $\mathrm{B}$, the position effectively corresponds to the time delay of a square wave with a period of 8 pattern pairs.

One advantage of sequence B compared to sequence A is that it has been experimentally shown to have a $2 \mathrm{~dB}$ better average SNR and up to $4.7 \mathrm{~dB}$ better SNR when comparing extreme cases. More important for the application in autonomous system control, however, is the behavior in case of an erroneous detection. Sequence A is composed in a binary search fashion and therefore a single bit error in the received optical waveform can lead to an error of up to half the array size in the determined position. The possibility of such jumps is a significant challenge for the control mechanism for position alignment. On the other hand, in the case of sequence $B$, the position error induced by a single bit error cannot exceed 1 pixel for all but the boundary pixels.

\section{A ROBOT CONTROLLED BY SMART LIGHTING}

A prototype autonomous device with control via visible light is realized by a silicon photodiode which is mounted

on two linear motion stages in the $x$ and $y$ directions. Each motion stage is moved by a direct-current (DC) motor which in turn is controlled by an Arduino Uno micro-chip board. The same micro-controller also samples the optical signal received by the photodiode and decodes the transmitted information. A photograph of the setup is shown in Fig. 1b.

When the LED array transmits the positioning pattern sequence, the Arduino determines the position of the photodiode from the received optical signal. This value is then used as the input to a proportional-integral-derivative (PID) control loop that drives the DC motors in order to move the photodiode to a given target position. It is indeed observed that when using pattern sequence type A, occasional bit errors are a significant problem for the reliability of the operation of the PID control loop. Therefore, careful choice of the pattern sequence is crucial for this application.

The target position itself can also be sent to the device via a sequence of illumination patterns. To enable this, we use a sequence of 36 patterns in total for operation on a $16 \times 16$ grid. 32 patterns are used to provide positioning and transmission of the target position, 2 additional patterns are used to provide an embedded clock signal and 2 further patterns are included to distinguish between positioning mode and transmission of the target position. In principle, any other control signal in addition to the target position can also be sent in the same fashion, thus enabling full wireless optical control.

\section{CONCLUSION}

Control of automated devices based entirely on visible light illumination is possible using structured illumination from LED-arrays. Careful choice of the illumination patterns used for this approach is crucial for reliable operation. This technology may find use e.g. for robot control in warehouses and automated manufacturing. With appropriate array scaling and design of relay optics, such control should be achievable over areas of square meters.

\section{ACKNOWLEDGMENT}

The authors thank the Engineering and Physical Sciences Research Council for funding under the grant EP/M01326X/1, QuantIC. There are no EPSRC-related datasets associated with this publication.

\section{REFERENCES}

[1] S. Zhang et al., "1.5 Gbit/s Multi-Channel Visible Light Communications Using CMOS-Controlled GaN-Based LEDs," J. Lightwave Technol., vol. 31, no. 9, pp. 1211-1216, 2013.

[2] J. Herrnsdorf et al., "Fast and accurate positioning system enabled by structured illumination with light-emitting diodes," submitted to J. Lightwave Technol.

[3] S. Rajbhandari et al., "High-Speed Integrated Visible Light Communication System: Device Constraints and Design Considerations," IEEE J. Sel. Areas Comm., vol. 33, no. 9, pp. 1750-1757, 2015.

[4] J. J. D. McKendry et al., "Individually Addressable AlInGaN Micro-LED Arrays With CMOS Control and Subnanosecond Output Pulses," IEEE Photon. Technol. Lett., vol. 21, no. 12, pp. 811-813, 2009.

[5] Z. J. Liu et al., "Monolithic LED Microdisplay on Active Matrix Substrate Using Flip-Chip Technology," J. Sel. Topics Quantum Electron., vol. 15, no. 4, pp. 1298-1302, 2009.

[6] J. Herrnsdorf et al., "Concept of a GaN-LED-based positioning system using structured illumination," in IEEE Photonics Conference, 2015. 